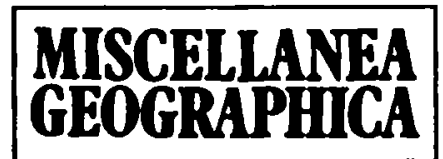

WARSZAWA 1992 Vol. 5

Jerzy Boryczka

\title{
THE INFLUENCE OF PARAMETERS OF THE SOLAR SYSTEM ON EARTH'S CLIMATE
}

\section{INTRODUCTION}

The paper presents an effort aimed at identification of causes of natural changes of climate from the 18 th through 21 st century.

The influence of parameters of the solar system (SS) on Sun's activity (Sun spot numbers) and - directly or indirectly - on Earth's climate was analysed.

In order to do this the spectra of oscillations of the solar system (1749-1980), Wolf's numbers (1749-1980), air temperatures in Warsaw (1779-1979), Cracow (1826-1980), Prague (1771-1980), Central England (1659-1973) and Colombo (1859-1980), as well as precipitation in Warsaw (1813-1980), Cracow (18761980) and Colombo (1869-1890) were compared.

The spectra of oscillations and the true periods of the variables analysed were determined using the method of J.Boryczka: according to the consecutive regression sinusoids.

Heliocentrical ecliptic coordinates of planets (Table 1) were determined from empirical formulae published by A.P.Reznikov (1982) for geophysical purposes.

Table 1

Planets of the solar system, their masses and periods of revolution arounc Sun

\begin{tabular}{|l|c|c|}
\hline \multicolumn{1}{|c|}{ Planets } & Mass & Revolution period \\
\hline Mercury & 0.055 & 0.241 \\
\hline Venus & 0.815 & 0.615 \\
\hline Earth & 1.000 & 1.000 \\
\hline Mars & 0.107 & 1.881 \\
\hline Jupiter & 317.820 & 11.862 \\
\hline Satum & 95.280 & 29.458 \\
\hline Uranus & 14.560 & 84.015 \\
\hline Neptune & 17.280 & 164.790 \\
\hline Pluto & 0.110 & 250.000 \\
\hline
\end{tabular}


The parameters of the solar system were defined as follows:

F - the resultant gravitational force of planets: $\vec{F}=\sum_{i} \frac{m_{i}}{r_{i}^{3}} \cdot \overrightarrow{r_{i}}$,

I - the total momentum of planets: $\overrightarrow{I=} \sum_{i} m_{i} \overrightarrow{r_{i}} \times \overrightarrow{r_{i}}$,

$\mathrm{R}$ - distance of the mass centre of SS from the Sun: $\vec{R}=[X, Y, Z]=\sum_{i} m_{i} \overrightarrow{r_{i}}$

$\ddot{k}$ - radial acceleration of the Sun,

$\mathrm{Z}$ - distance from the mass centre of SS to the ecliptic plane

$\cos \Gamma-$ cosine of the angle between the radius vectors of the mass centre, $\vec{R}$, and the geometrical centre, $\vec{r}=\frac{1}{n} \sum_{i} \overrightarrow{r_{i}}$, where $m_{i}, \overrightarrow{r_{i}}$, are, respectively, mass and radius vector of an $i$-th planet and $M$ is the mass of the solar system.

Negative results obtained by A.Schuster (1911) with regard to correlation between the gravitation forces of Jupiter, Venus, Mercury and the numbers of Sun spots slowed down significantly the research on tides and turbulences on the Sun.

\section{THE METHOD OF FINDING SPECTRA OF OSCILLATIONS AND TRUE PERIODS}

The chronological series $y_{1}, \ldots, y_{n}$ of moving 12-month (consecutive) averages was approximated by the successive regression sinusoids:

$$
y=a_{o}+b \cdot \sin \left(\frac{2 \pi}{\Theta} t+c\right)
$$

by assuming periods: $\Theta=\frac{1}{12}, \frac{2}{12}, \ldots, N \quad(N=3000)$.

Parameters of the sinusoids, i.e. amplitude $\sigma$ and phase delay $c$ were determined from the least squares condition. Having introduced transformation $a_{1}=b \cdot \cos c, a_{2}=b \cdot \sin c$ we can treat equation of the sinusoid (1) as the equation of a regression plane with respect to variables

$x_{1}=\sin \omega t, x_{2}=\cos \omega t$

$y=a_{o}+a_{1} x_{1}+a_{2} x_{2}$

where $\omega=\frac{2 \pi}{\Theta}$ is the frequency of oscillations.

Amplitude $b$ and phase delay $c$ are calculated from the formulae

$$
b=\sqrt{a_{1}^{2}+a_{2}^{2}} \text { and } \operatorname{tg} c=\frac{\alpha_{2}}{\alpha_{1}}
$$


The sequence of amplitudes $b_{1}, \ldots b_{N}$ constitutes the empirical spectrum of oscillations, while $c_{1}, \ldots, c_{N}-$ the vector of phase delays.

Local maxima of the spectrum of oscillations - of the sequence of amplitudes $b(\Theta)$ are the periods $\Theta_{r}$ and frequencies $\omega_{r}=\frac{2 \pi}{\Theta_{r}}$.

Verification of the true periods was performed through determination of the oscillogram of correlations - the sequence of coefficients of multiple correlation: $\rho_{1}, \ldots \rho_{N}$ Fisher-Snedecor test with 2 and $n-3$ degrees of freedom was applied.

Harmonic analysis (Fourier series), in view of fictitious periods $n, \frac{n}{2}, \frac{n}{3}, \ldots$ and spectral analysis (autocorrelation), in view of its limitation ot short periods, $<\frac{n}{2}$, are not adequate for analysing periodicity of climate changes.

\section{SIMILARITY OF SPECTRA OF OSCILLATIONS OF SOLAR ACTIVITY AND OF PARAMETERS OF THE SOLAR SYSTEM}

Comparison was made of the spectra of oscillations (moving averages for 12month periods) of solar activity and of parameters of the solar system in the period 1749-1980 $(n=2773)$, determined with the period step equal $\Delta \Theta=\frac{1}{12}$. Besides the true periods $(\Theta)$ and the amplitudes $(b)$, the values of multiple correlation coefficient $\rho$ are given here (Table 2).

All the true periods of solar activity appear in the spectra of oscillations of parameters of the solar system.

\begin{tabular}{|c|c|c|c|c|c|c|c|}
\hline \multicolumn{2}{|c|}{ Wolf's number } & \multicolumn{2}{c|}{ Parameters of SS } & \multicolumn{2}{c|}{ Wolf's number } & \multicolumn{2}{c|}{ Parameters of SS } \\
\hline$\Theta$ & $\rho$ & $\Theta$ & $\rho$ & $\Theta$ & $\rho$ & $\Theta$ & $\rho$ \\
\hline 7.42 & 0.11 & 7.75 & $0.25 R$ & 14.83 & 0.12 & 14.75 & $0.39 \mathrm{I}$ \\
\hline 8.08 & 0.13 & 7.83 & $0.14 \Gamma$ & 17.33 & 0.07 & 17.58 & $0.18 \mathrm{R}$ \\
\hline 8.50 & 0.15 & 8.50 & $0.13 \Gamma$ & 19.08 & 0.05 & 19.83 & $0.81 \mathrm{R}$ \\
\hline 9.42 & 0.28 & 9.33 & $0.13 \mathrm{~F}$ & 21.25 & 0.13 & 22.67 & $0.16 \mathrm{R}$ \\
\hline 9.92 & 0.43 & 9.92 & $0.12 \mathrm{~F}$ & 28.75 & 0.10 & 29.50 & $0.68 \mathrm{Z}$ \\
\hline 11.08 & 0.50 & 11.25 & $0.69 \Gamma$ & 43.17 & 0.14 & 45.33 & $0.31 \Gamma$ \\
\hline 12.00 & 0.23 & 11.83 & $0.65 \mathrm{Z}$ & 58.75 & 0.23 & 58.83 & $0.06 \mathrm{R}$ \\
\hline 12.92 & 0.14 & 12.75 & $0.42 \mathrm{R}$ & 90.75 & 0.33 & 82.75 & $0.12 \mathrm{I}$ \\
\hline 13.92 & 0.12 & 13.92 & $0.23 \mathrm{R}$ & 179.00 & 0.18 & 185.17 & $0.15 \Gamma$ \\
\hline
\end{tabular}

There is little probability that the agreement of oscillation spectra -18 bands of frequencies of changes in solar activity and parameters of the solar system - is a purely random occurrence. 


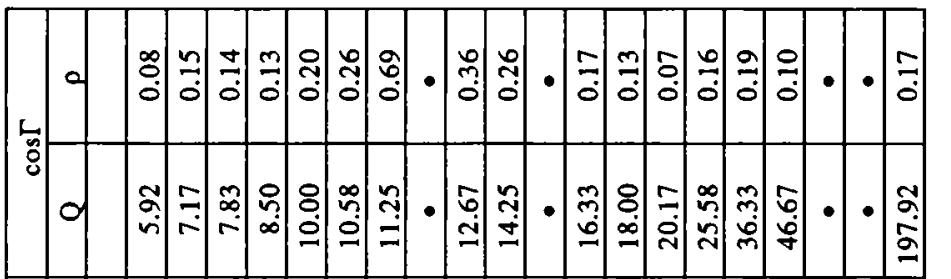

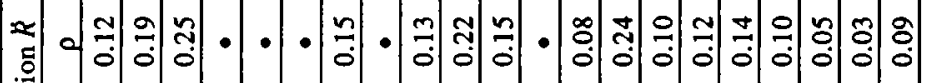

要

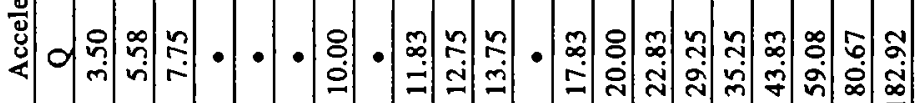

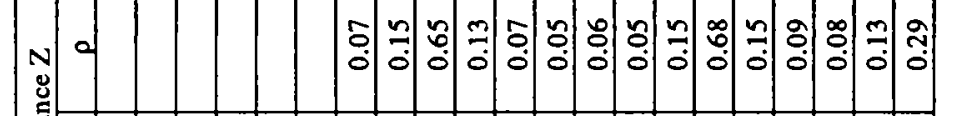

紊

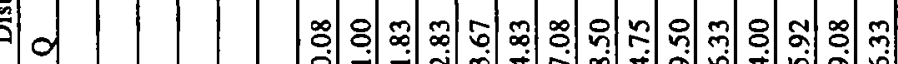

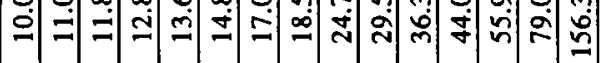

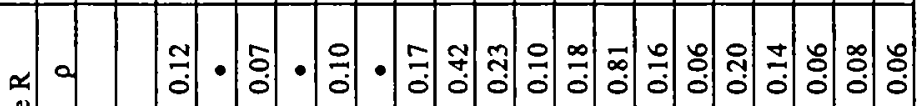

苛

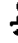

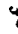

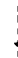

$=$

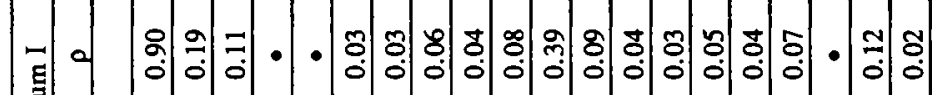

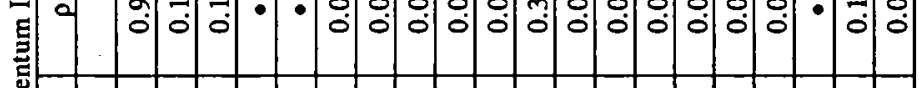

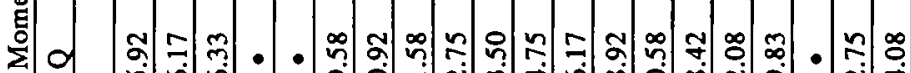

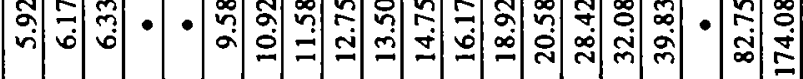

ะ

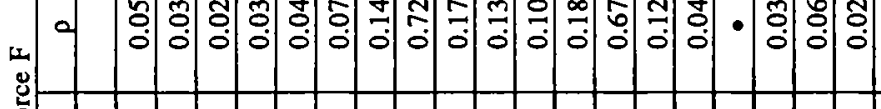

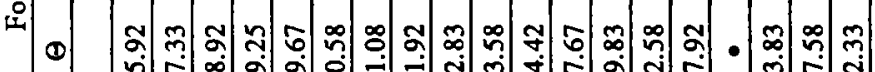

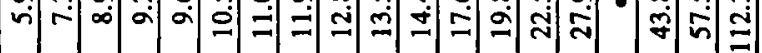

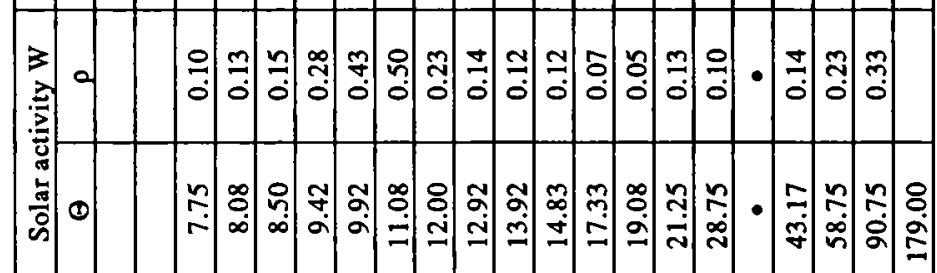


Almost all periods $\Theta$ (amplitudes $b$ ) are significant at the confidence levels of $99 \%, 95 \%$, and $90 \%$. Critical values of multiple correlation coefficient, according to Fisher-Snedecor test with 2 and 2270 degrees of freedom are, respectively, $0.058,0.047$ and 0.041 .

Two strongest cycles deserve special attention: 11.08 years for solar acitivity and 11.25 years for cosine of $\Gamma$ angle (angle between radius vectors of the mass center and the geometrical centre). They are desribed with the equations of regression sinusoids:

$$
\begin{array}{ll}
W=a_{o}+28.3 \cdot \sin \left(\frac{2 \pi}{11.08} t+1.6579\right) & \rho=0.499 \\
\cos \Gamma=a_{o}+0.64 \cdot \sin \left(\frac{2 \pi}{11.25} t+2.680\right) & \rho=0.685
\end{array}
$$

where $W$ denotes solar activity.

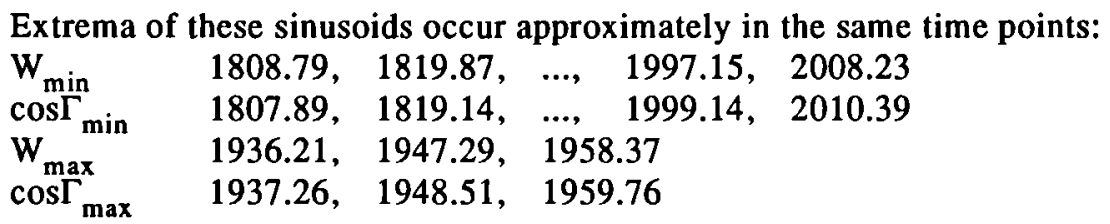

Minima of solar spots occur in the years in which the mass centre of the solar system and its geometric centre are located on a straight line on the opposite sides of the Sun $(\cos \Gamma=-1)$. On the other hand, maxima of Sun spots (absolute maximum in 1957) occur when these two centres are on the same side of the Sun $(\cos \Gamma=1)$.

In such situations the action of gravitational forces of the planets and the dynamic effects overlap, which is also confirmed by the coincidence of the strongest cycles of the gravitational force $F$ and the distance of the mass centre $R$.

THE INFLUENCE OF SOLAR ACTIVITY UPON EARTH'S CLIMATE

There is also a great similarity between the spectra of oscillation of climate, as expressed by air temperature and precipitation, as well as solar activity (Table 3 and 4 , Figs. 1 and 2 ).

It is interesting to note that the strongest cycle of 7.75 years for air temperature in Warsaw, Cracow, Prague, Central England and Colombo is the same as the strongest cycle of acceleration $k$ of the Sun with respect to the mass centre of the solar system, and is close in value to two cycles: 7.42 years and 7.75 years, of solar activity.

The equations of regression sinusoids are for this particular cycle as follows: 
Table 3

True periods of air temperature in Warsaw (1779-1979), Cracow (1826-1986), Prague (1771-1980), Central England (1659-1973) and Colombo (1869-1980), together with amplitudes $b$ of variations

\begin{tabular}{|c|c|c|c|c|c|c|c|c|c|}
\hline \multicolumn{2}{|c|}{ Warsaw } & \multicolumn{2}{c|}{ Cracow } & \multicolumn{2}{c|}{ Prague } & \multicolumn{2}{c|}{ England } & \multicolumn{2}{c|}{ Colombo } \\
\hline$\Theta$ & $b$ & $\Theta$ & $b$ & $\Theta$ & $b$ & $\Theta$ & $b$ & $\Theta$ & $b$ \\
\hline 3.42 & 0.23 & 3.17 & 0.19 & 3.42 & 0.18 & 3.42 & 0.06 & 3.33 & 0.06 \\
\hline 5.50 & 0.19 & 5.50 & 0.20 & 5.42 & 0.16 & 5.17 & 0.13 & 5.25 & 0.07 \\
\hline 6.50 & 0.18 & 6.50 & 0.21 & 6.50 & 0.14 & 6.33 & 0.10 & 6.50 & 0.08 \\
\hline 7.75 & 0.28 & 7.75 & 0.29 & 7.75 & 0.25 & 7.33 & 0.14 & 7.58 & 0.05 \\
\hline 11.17 & 0.15 & 11.42 & 0.21 & 10.33 & 0.20 & 11.17 & 0.11 & 10.17 & 0.10 \\
\hline 12.83 & 0.21 & 12.50 & 0.19 & 14.17 & 0.25 & 12.50 & 0.13 & 13.00 & 0.07 \\
\hline 17.50 & 0.19 & 16.58 & 0.24 & 17.75 & 0.21 & 17.83 & 0.10 & 16.75 & 0.07 \\
\hline 21.75 & 0.12 & 22.00 & 0.07 & 22.25 & 0.18 & 23.58 & 0.21 & 23.17 & 0.18 \\
\hline 28.50 & 0.07 & 31.67 & 0.14 & 29.42 & 0.14 & 35.08 & 0.10 & 36.17 & 0.15 \\
\hline 40.92 & 0.10 & 54.58 & 0.21 & 51.25 & 0.22 & 58.50 & 0.11 & $\bullet$ & $\bullet$ \\
\hline 89.67 & 0.21 & 82.83 & 0.23 & 80.33 & 0.22 & 73.42 & 0.17 & 86.42 & 0.26 \\
\hline 195.17 & 0.56 & & & 160.33 & 0.47 & 102.75 & 0.25 & & \\
\hline
\end{tabular}

Table 4

True periods of precipitation in Warsaw (1813-1980), Cracow (1876-1980) and Colombo (1869-1980), together with amplitudes $b$

\begin{tabular}{|c|c|c|c|c|c|}
\hline \multicolumn{2}{|c|}{ Warsaw } & \multicolumn{2}{c|}{ Cracow } & \multicolumn{2}{c|}{ Colombo } \\
\hline$\Theta$ & $b$ & $\Theta$ & $b$ & $\Theta$ & $b$ \\
\hline 3.50 & 31.6 & 3.42 & 35.3 & 3.42 & 182.4 \\
\hline 4.25 & 36.4 & 3.67 & 28.6 & 4.17 & 145.2 \\
\hline 5.33 & 38.4 & 5.33 & 19.7 & 5.83 & 105.2 \\
\hline 6.00 & 31.3 & 5.92 & 30.0 & 7.08 & 122.4 \\
\hline 9.67 & 24.2 & 10.33 & 31.9 & 7.67 & 130.8 \\
\hline 11.67 & 26.6 & 12.25 & 33.5 & 12.17 & 18.36 \\
\hline 16.33 & 38.0 & 14.00 & 35.0 & 16.50 & 134.4 \\
\hline 19.33 & 33.7 & 19.25 & 15.5 & $\bullet$ & $\bullet$ \\
\hline 22.25 & 22.1 & 23.08 & 32.5 & 25.00 & 99.4 \\
\hline 28.40 & 16.8 & 29.58 & 54.7 & $\bullet$ & $\bullet$ \\
\hline 39.33 & 16.8 & $\bullet$ & $\bullet$ & 42.00 & 268.8 \\
\hline 56.42 & 30.2 & 62.33 & 44.9 & $\bullet$ & $\bullet$ \\
\hline 112.50 & 28.8 & $\bullet$ & $\bullet$ & 109.75 & 240.0 \\
\hline
\end{tabular}



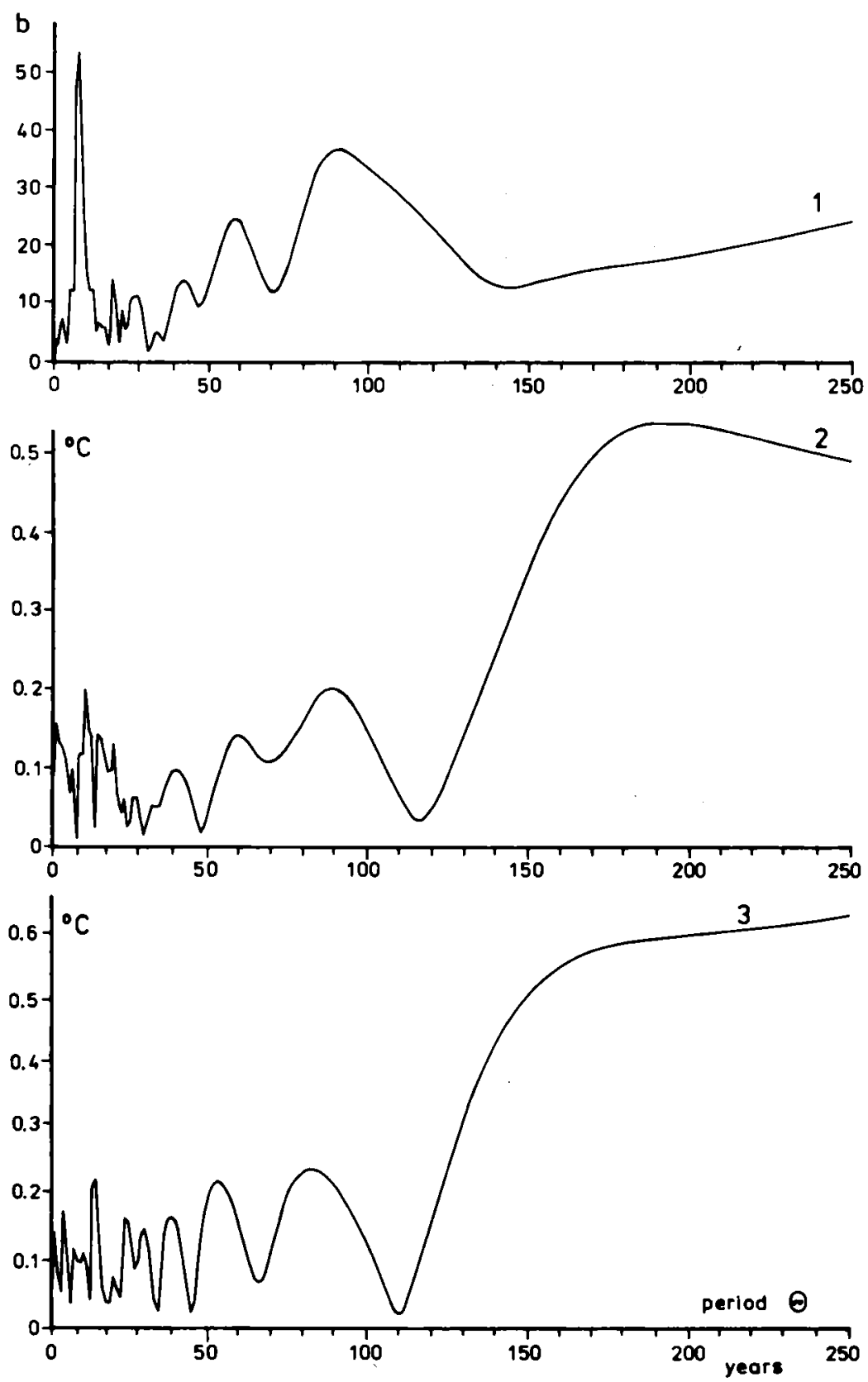

Fig. 1. Spectra of oscillations of annual averages for: 1 - Wolf's number (1749-1980), 2 - air temperatures in Warsaw (1779-1979) and 3 - air temperatures in Cracow (1826-1985) 


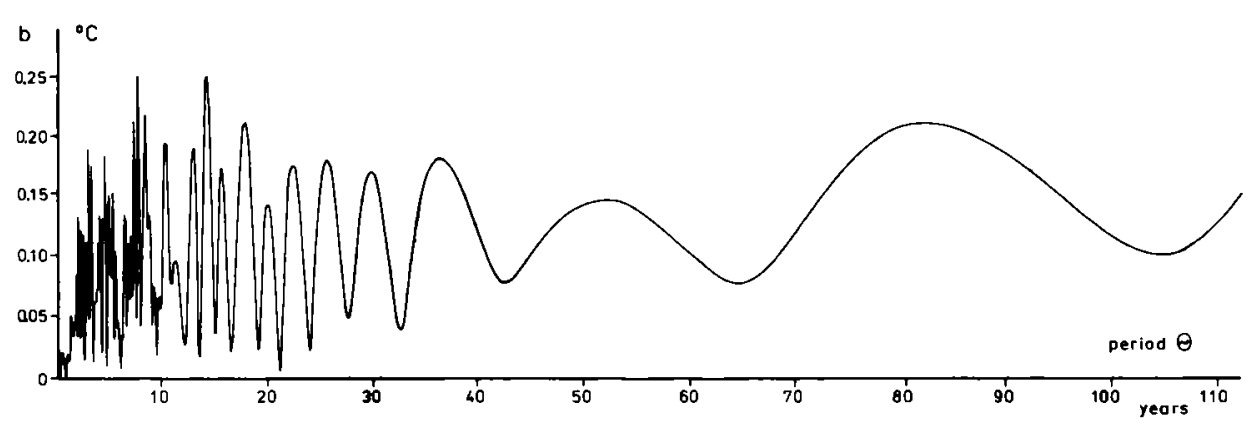

Fig. 2. Spectra of oscillations of averages of consecutive 12-month series of air temperatures in Prague (1771-1980)

$$
\begin{array}{ll}
\Delta W=5.510 \cdot \sin \left(\frac{2 \pi}{7.75} t-3.136\right) & \text { (Wolf numbers), } \\
\Delta k=1.130 \cdot \sin \left(\frac{2 \pi}{7.75} t+1.21\right) & \text { (parameter } k \text { ) } \\
\Delta T=0.282 \cdot \sin \left(\frac{2 \pi}{7.75} t+0.147\right) & \text { (Warsaw), } \\
\Delta T=0.290 \cdot \sin \left(\frac{2 \pi}{7.75} t+0.541\right) & \text { (Cracow), } \\
\Delta T=0.253 \cdot \sin \left(\frac{2 \pi}{7.75} t-0.019\right) & \text { (Prague). }
\end{array}
$$

The range of temperature changes in this cycle is $0.56^{\circ} \mathrm{C}$ for Warsaw, $0.58^{\circ} \mathrm{C}$ for Cracow and $0.50^{\circ}$ for Prague.

These cycles are synchronous. Their maxima appear in the same time, in spite of varying lengths of chronological sequences. Some examples for the coincidence of maxima are given below:

$\begin{array}{lllll}\text { Solar activity } & R \text { parameter } & \text { Warsaw } & \text { Cracow } & \text { Prague } \\ 1932.06 & 1934.44 & 1934.76 & 1934.77 & 1934.71 \\ 1939.80 & 1942.19 & 1942.51 & 1942.52 & 1942.46 \\ 1947.56 & 1949.94 & 1950.26 & 1950.27 & 1950.21 \\ 1955.31 & 1957.69 & 1958.01 & 1958.02 & 1957.96\end{array}$

This confirms the proposition of K.P.Butusov (1992) that solar spots are caused by turbulences in the Sun resulting from various accelerations of its core and external layers in their movement around the centre of mass of the solar system. 
There is also a statistically significant 11 -year cycle of air temperature, namely:

Warsaw: $\quad \Delta T=0.148 \cdot \sin \left(\frac{2 \pi}{11.17} t+0.731\right)$,

Cracow: $\quad \Delta T=0.206 \cdot \sin \left(\frac{2 \pi}{11.42} t-2.86\right)$,

Prague: $\quad \Delta T=0.100 \cdot \sin \left(\frac{2 \pi}{11.25} t-2.26\right)$,

England: $\quad \Delta T=0.106 \cdot \sin \left(\frac{2 \pi}{11.17} t+2.82\right)$.

which is close to the 11.08-year cycle of solar activity and to the 11.25 -year cycle of $\cos \Gamma$.

Air temperature undergoes changes over longer time periods, too well:

Warsaw: $\quad \Delta T=0.207 \cdot \sin \left(\frac{2 \pi}{89.67} t+2.479\right)$,

Cracow: $\Delta T=0.229 \cdot \sin \left(\frac{2 \pi}{82.83} t-3.12\right)$,

Prague: $\quad \Delta T=0.215 \cdot \sin \left(\frac{2 \pi}{80.92} t-0.875\right)$,

England: $\Delta T=0.254 \cdot \sin \left(\frac{2 \pi}{102.75} t+3.04\right)$,

Colombo: $\Delta T=0.256 \cdot \sin \left(\frac{2 \pi}{86.42} t-0.569\right)$.

Air temperature changes over these cycles were contained in the intervals of $0.41^{\circ} \mathrm{C}$ for Warsaw, $0.46^{\circ} \mathrm{C}$ for Cracow, $0.43^{\circ} \mathrm{C}$ for Prague, $0.51^{\circ} \mathrm{C}$ for England and also $0.51^{\circ} \mathrm{C}$ for Colombo.

These periods of air temperature changes for Warsaw - 89.67 years, and Colombo - 86.42 years, are the closest to the period of secular cycle of solar activity, i.e. 90.75 years:

$$
\Delta W=19.0 \cdot \sin \left(\frac{2 \pi}{90.75} t+0.0128\right)
$$

Minimum air temperatures in Warsaw correspond to minima of secular Sun spot changes. In Colombo, on the other hand, near to the equator, the situation is 
opposite: temperature minima correspond to maxima of solar activity in the secular cycles:

Solar activity $\left(W_{\min }\right): 1724.63,1815.88,1906.63,1996.88,2087.63$

Warszaw $\left(T_{\min }\right): \quad 1720.21,1809.88,1899.55, \quad 1989.22,2078.89$

Colombo $\left(T_{\max }^{\min }\right): \quad 1724.59,1811.01,1897.43,1983.85,2070.27$

On the other hand, periods of air temperature changes in this cycle for Cacow -82.83 years, and for Pague -80.92 years, are similar to the period of secular cycle of variations of distance $Z$ of the mass centre of the solar system from the ecliptic plane -79.08 years:

$$
\Delta Z=2.210 \cdot \sin \left(\frac{2 \pi}{79.08} t-2.16\right)
$$

Secular temperature minima in Prague appear more or less simultaneously with the minima of distance of the mass centre. $Z$ :

Distance $Z_{\min }: \quad 1755.42, \quad 1834.50, \quad 1913.58, \quad 1992.66, \quad 2071.74$

Prague $\mathrm{T}_{\min }: \quad 1761.01,1841.96,1922.88,2003.80,2084.72$.

There also exists a nearly two-secular period of temperature cycle in Warsaw (191.17 years), similar to the periods of parameters of the solar system, $\cos \Gamma$ $=197.92$ years, and of solar activity, 179.0 years.

The shortest periods of cycles related to air temperature are: in Warsaw 3.42 , 5.50 and 6.50 years; in Cracow 3.17, 5.50 and 6.50 years; in Prague 3.42, 5.42 and 6.50 years; in England 3.42, 5.17 and 6.33 years; and in Colombo 3.33, 5.25 and 6.50 years. These periods do not appear in the spectrum of solar activity, but in the spectrum of solar acceleration, $k: 3.50,4.00$ and 5.58 years.

This short periodical variability applies also to precipitation: in Warsaw $3.50,4.25$ and 5.33 years; in Cracow $-3.42,3.67$ and 5.33 years; and in Colombo $-3.42,4.17,5.83$ years.

Another statistically significant cycle is the 11-year cycle of precipitation, whose exact periods are: in Warsaw - 11.67 years, in Cracow -12.25 , and in Colombo - 12.17 years. This cycle is similar to the cycles of: solar activity 11.08 years, distance of the mass cent of the solar system from the ecliptic $(Z)-$ 11.83 years, $\rho=0.65$, and the parameter $\cos \Gamma-11.25$ years, $\rho=0.69$. (Note also that $\mathbf{1 1 . 8 6 2}$ years is the period of Jupiter's revolution around Sun.)

Apart from those mentioned already there also exists a cycle of some 30-year period, concerning precipitation, having important amplitude. The periods of this cycle in Warsaw and Cracow are, respectively: 28.000 years and 29.58 years. Analogous periodicity applies to the distance of the mass centre of the solar system from the ecliptic -29.50 years and to solar activity -28.75 years: 
Distance Z:

$$
\Delta W=11.60 \sin \left(\frac{2 \pi}{29.50} t t 2.02\right)
$$

Wolf numbers:

$$
\Delta W=5.64 \sin \left(\frac{2 \pi}{28.75} t t 0.857\right)
$$

Cracow:

$$
\Delta P=54.72 \sin \left(\frac{2 \pi}{29.58} t t 2.14\right) \text {, }
$$

Warsaw:

$$
\Delta P=16.80 \sin \left(\frac{2 \pi}{28.00} t-1.330\right),
$$

It is interesting to note that the strongest precipitation cycle in Cracow (29.58 years, with amplitude of $2 b=109.4 \mathrm{~mm}, \rho=0.68$ ) coincides with the strongest cycle (period of 29.50 years, $\rho=0.68$ ) of the distance of the mass centre of the solar system from the ecliptic (Fig.3).

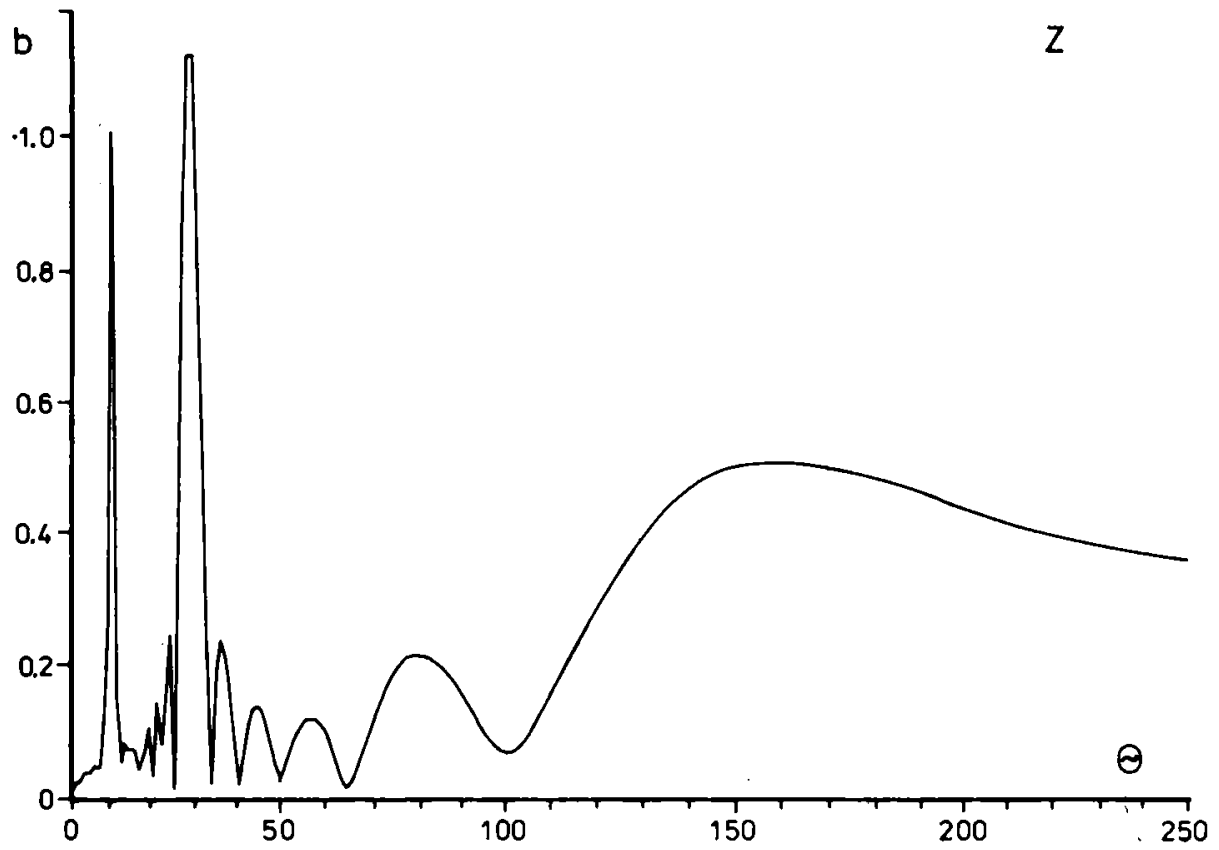

Fig. 3. Spectrum of oscillation of distance $Z$ from the mass centre of $S S$ to the ecliptic plane. 
Precipitation maxima in the 30-year cycle occur close in time to the minima of distances between the mass centre of the solar system and the ecliptic and to the minima of solar activity, for example in the following time instances:

Distance $\left(\mathrm{Z}_{\min }\right): \quad 1908.14, \quad 1937.64,1967.14,1996.64,2026.14$, Wolf numbers $\left(\mathrm{W}_{\min }\right): 1909.39,1938.14,1966.89,1995.64,2024.39$, Cracow $\left(\mathrm{P}_{\max }\right)$ : $\quad 1901.90,1931.48,1961.06,1990.64,2020.22$, Warsaw $\left(\mathrm{P}_{\max }\right): \quad 1908.93,1936.93,1964.93,1992.93,2020.93$.

The agreement of the main bands of frequencies of precipitation oscillations with the spectra of oscillations of parameters of the solar system (better than for solar activity) indicates a witness of a direct - gravitational - influence of the planet an system on the Earth's atmosphere.

\section{REFERENCES}

B ory czka J., 1990 "Changes in Earth's climate in the 18th through 21 st centuries and their reasons", Miscellanea Geographica.

B u t u s ow K.P., 1972, "K voprosu o tsiklichnosti sol'nechnoi aktivnosti" (On the question of periodicity of solar activity), in: Sol'ntse, elektichestvo zhizni, MGU, Moskva.

S chuster A., 1911, "The influence of Planets on the formation of Sunspots), Proc.Roy.Soc. Ser. A. vol. 85, NA 575, Math.cal. phys. sci.

Reznik ov A.P., 1982 , Przedskazan' ie iestestvennykh protsesosov obuchaiushcheisia sisitemoi (Prediction of natural processes by a learning system), Novosibirsk. 